\title{
The CMS Level-1 Endcap Muon Trigger at the High-Luminosity LHC
}

\section{Daniel Guerrero ${ }^{\dagger, *}$}

University of Florida,

Gainesville, Florida, United States

E-mail: daniel.guerrero@cern.ch

The Phase-2 upgrade of the CMS Level-1 (L1) trigger will be carried out to achieve the highest possible acceptance for physics processes in the large pile-up and instantaneous luminosity conditions of the High-Luminosity Large Hadron Collider (HL-LHC). In the CMS endcap region, muon reconstruction in the L1 trigger is complicated by the non-uniform magnetic field, high pileup and punch-through interactions. However, the new muon detectors and the upgraded trigger processing capabilities proposed for the Phase- 2 upgrade will allow the implementation of novel techniques to address successfully these challenges. For instance, track-finding and reconstruction of the standalone and displaced muons are performed by a neural network-based algorithm. In addition, a proposed Global Muon Trigger system will have access to tracker tracks, muon trigger tracks and individual muon detector hits. These objects can then be combined to improve the muon momentum resolution, reduce the muon trigger rates, and form multi-object triggers targeting specific processes such as charged lepton flavour violating $\tau \rightarrow 3 \mu$ decays. We present here preliminary studies addressing all these new capabilities.

40th International Conference on High Energy physics - ICHEP2020

July 28 - August 6, 2020

Prague, Czech Republic (virtual meeting)

\footnotetext{
* Speaker

${ }^{\dagger}$ On behalf of the CMS Collaboration
} 


\section{Introduction}

A new territory of experimental high energy physics will be explored during the operation of the ultimate configuration of the Large Hadron Collider (LHC), denominated as the High-Luminosity LHC (HL-LHC). This accelerator will produce proton-proton (pp) collisions at $14 \mathrm{TeV}$ of centerof-mass energy $(\sqrt{\mathrm{s}})$, with a peak of instantaneous luminosity of $7.5 \times 10^{34} \mathrm{~cm}^{-2} \mathrm{~s}^{-1}$, and an average of around 200 pile-up (PU) interactions. The Phase-1 of the LHC operation is ongoing and will continue until 2024. Then, the CMS experiment [1] will undergo its Phase-2 upgrade [2], which will feature new detectors, trigger and data acquisition systems to overcome the HL-LHC challenging data-taking conditions, enhance selectivity for a variety of physics processes and fully exploit the large dataset of about $4000 \mathrm{fb}^{-1}$ of integrated luminosity.

As part of the Phase-2 upgrade, the muon system will add new detectors to extend the high pseudorapidity $(\eta)$ region coverage up to $|\eta|=2.8$, will improve its electronics, and will send more accurate information from individual muon hits (stubs) to the Level-1 (L1) trigger [3]. Similarly, the L1 trigger upgrade will lead to a $12.5 \mu$ s latency, a maximum $750 \mathrm{kHz}$ output rate, state-of-the-art electronics (e.g. FPGAs equipped with $28 \mathrm{~Gb} / \mathrm{s}$ transceivers) and a flexible architecture, which will enable innovative and complex algorithms to be implemented. The L1 trigger will introduce a new correlator layer where objects from different processing paths (muon system, calorimeters, tracking and particle flow techniques) can be combined to generate sophisticated triggers [4]. This paper focuses on the algorithms, performance and prospects of the L1 endcap muon trigger.

\section{Triggering on endcap muons at the HL-LHC}

An efficient online selection of muons coming from processes with broad kinematic range will be fundamental for the HL-LHC physics program. At the L1 trigger, a measurement of the muon transverse momentum $\left(\mathrm{p}_{\mathrm{T}}\right)$ with excellent resolution helps to control the rate and keep low trigger thresholds. The endcap standalone prompt muon reconstruction is challenging due to the complex detector geometry, non-uniform magnetic field, high PU, and punch-through interactions. In Phase-1 [5], the L1 endcap muon track finder algorithm, EMTF, reconstructs muon tracks and their kinematics by identifying stubs compatible with a muon track via pattern recognition and then a boosted decision tree (BDT) uses their information to assign the expected $\mathrm{p}_{\mathrm{T}}$ value. This BDT prediction scheme is encoded in a Look-Up Table (LUT), and thus the performance is limited by the number of addresses and compression of the inputs.

The Phase-2 algorithm, EMTF++, will benefit from the extensive stub information (position, type, time, quality, and bend angle) of the new detectors. The total number of predefined patterns will increase more than tenfold with respect to EMTF and the $\mathrm{p}_{\mathrm{T}}$-assignment will be performed using a neural network $(\mathrm{NN})$. The preliminary studies on simulation show that the EMTF++ performance will surpass the one of the EMTF. For example, under the $200 \mathrm{PU}$ scenario, the EMTF++ rate at a 20 $\mathrm{GeV} \mathrm{L1} \mathrm{p}_{\mathrm{T}}$ threshold is expected to be around $20 \mathrm{kHz}$, which is 2.5 times lower with respect to the EMTF. There is also an efficiency improvement, as presented in Figure 1(left). A similar method will allow for the track-finding and reconstruction of displaced muons to be implemented [4].

The Phase-2 Global Muon Trigger (GMT) will have the ability to create new types of muon objects using information from muon tracks, muon stubs, and fully reconstructed tracks from the track finder (up to $|\eta|<2.4$ ). It will be able to generate track-correlated muon objects by matching tracker tracks to standalone muon tracks or individual muon stubs. The procedure is performed via 
$\mathrm{p}_{\mathrm{T}}$-dependent $\Delta \phi / \Delta \eta$ matching windows that address the muon propagation across the detector and multiple scattering. These objects will significantly improve the $\mathrm{p}_{\mathrm{T}}$ resolution, and consequently, sharper efficiency curves and lower rates will be achieved as seen in Figure 1 (center) and (right), respectively. In addition, the GMT will create correlated multi-muon objects such as muon-jets. Preliminary studies of triggers using muon-jets with three objects (track+stub or stubs) show the improvement of signal efficiency for signatures with low- $\mathrm{p}_{\mathrm{T}}$ and forward muons. For instance, the signal acceptance of $\tau \rightarrow 3 \mu$ lepton-flavor violating decays is expected to increase by a factor of 5-10 with respect to Phase- 1 at a manageable rate, and there is still room for future improvements [4].
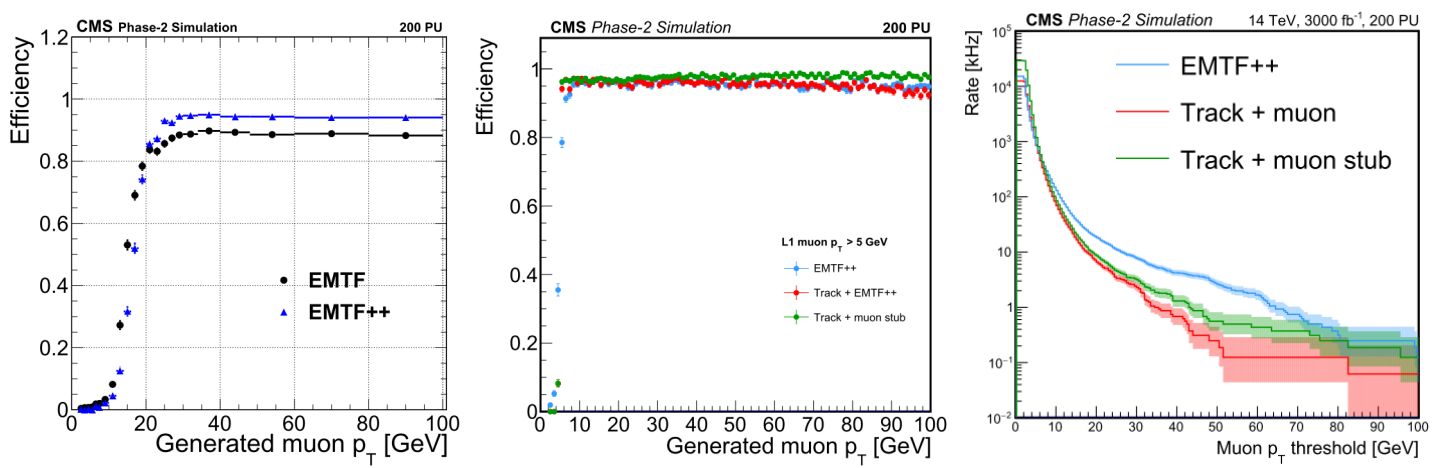

Figure 1: Phase-2 L1 endcap muon trigger performance [4]. Left: EMTF/EMTF++ efficiency vs muon $\mathrm{p}_{\mathrm{T}}$ (L1 muon $\mathrm{p}_{\mathrm{T}}>20 \mathrm{GeV}$ ), Center: Efficiency vs muon $\mathrm{p}_{\mathrm{T}}$, Right: Rate vs $\mathrm{L} 1 \mathrm{p}_{\mathrm{T}}$ threshold.

Tests of the EMTF++ and track+EMTF++ correlator algorithms in a hardware demonstrator setup using a Virtex-7 FPGA have been performed. The algorithms have been implemented in firmware. Particularly, the NN firmware is written in Vivado HLS using the hls4ml software [6]. The results show that these algorithms can be implemented within the resource constraints of the Phase-2 hardware, and their implementation has been successfully validated [4].

\section{Summary}

The CMS Phase-2 upgrades and the large HL-LHC dataset will allow for the exploration of a broad physics phase space to take place. In particular, the new and improved endcap muon trigger will be fundamental to enable this program. The feasibility of the new algorithms has been tested with the first hardware demonstrators. Software, firmware and hardware developments are ongoing.

\section{References}

[1] CMS Collaboration. The CMS Experiment at the CERN LHC. JINST, 3:S08004, 2008. doi : $10.1088 / 1748-0221 / 3 / 08 / S 08004$.

[2] CMS Collaboration. Technical Proposal for the Phase-II Upgrade of the CMS Detector. 2015. URL: https: //cds . cern. ch/record/2020886.

[3] CMS Collaboration. The Phase-2 Upgrade of the CMS Muon Detectors. Technical Design Report CMS-TDR-016, 2017. URL: https://cds . cern. ch/record/2283189.

[4] CMS Collaboration. The Phase-2 Upgrade of the CMS Level-1 Trigger. Technical Design Report CMS-TDR-021, 2020. URL: https: //cds. cern. ch/record/2714892.

[5] CMS Collaboration. CMS Technical Design Report for the Level-1 Trigger Upgrade. 2013. URL: https: //cds . cern. ch/record/1556311.

[6] J. Duarte et al. Fast inference of deep neural networks in FPGAs for particle physics. JINST, 13(07):P07027, 2018. arXiv: 1804 .06913, doi : 10 . 1088/1748-0221/13/07/P07027. 